\title{
Nächtliche Autofahrt: drei Stunden ohne Pause wirken wie 0,8 Promille
}

\author{
Beim nächtlichen Autofahren wirkt \\ Müdigkeit wie Alkohol: Nach drei \\ Stunden ohne Pause entspricht die \\ Aufmerksamkeit derjenigen eines \\ Betrunkenen mit 0,8 Promille.
}

- In einem Cross-Over-Design wurden 14 gesunde Probanden (männlich, mittleres Alter 23,4 \pm 1,7 Jahre, mittlere Fahrstrecke pro Jahr $14250 \pm 4660 \mathrm{~km}$ ) untersucht. Sie absolvierten jeweils drei unterschiedlich lange Fahrzeiten-Blöcke auf offener Straße bei Nacht. Die Fahrzeiten betrugen zwei Stunden $(03,00$ $5,00 \mathrm{~h})$, vier Stunden (01.00-5.00 h) und acht Stunden (21,00-5,00 h).

Als primärer Endpunkt und Messgröße einer zunehmenden Einschränkung der Fahrtüchtigkeit wurde die Standarddeviation bzw. -abweichung der Lateralposition (SDLP) des für die Messung mit Video ausgerüsteten Fahrzeugs in $\mathrm{cm}$ in der letzten Fahrstunde gemessen. Das Videosystem maß zehnmal pro Sekunde die Lateralposition des
Fahrzeugs vom rechten markierten Fahrbahnrand. Die Daten wurden dann verglichen mit bereits bekannten Effekten von Alkoholeinfluss auf die Fahrtüchtigkeit.

Die Seitenabweichungen des Fahrzeuges nahmen mit der Länge der Fahrzeit zu. Eine Nacht-Fahrzeit von zwei Stunden entsprach der Fahrtüchtigkeit wie bei einem Blutalkoholspiegel von 0,5 Promille, nach drei Stunden sogar 0,8 Promille. Diese Ergebnisse unterschieden sich signifikant. Zwischen vier und acht Stunden Fahrzeit ergaben sich allerdings keine weiteren signifikanten Unterschiede.

\section{Kommentar}

Die Studie belegt eindrucksvoll den Einfluss von zunehmender Ermüdung auf die Fahrtüchtigkeit bei längeren Nachtfahrten. Ermüdung hat hierbei denselben Effekt wie Alkoholeinfluss. Dieser Effekt tritt schon nach zwei Stunden Fahrzeit ein und erreicht nach drei Stunden ein Ausmaß, das einem Alko-
hol-Blutspiegel von 0,8 Promille entspricht. Hier stellt sich die Frage, ob diesem Umstand bereits genügend Rechnung getragen wird. Kann man das Autofahren, insbesondere bei Nacht, überhaupt noch der Selbsteinschätzung und Eigenverantwortung der Fahrer überlassen? Oder sollten nicht möglichst schnell die technisch bereits zur Verfügung stehenden Erkennungssysteme von Müdigkeit beim Autofahren in unsere Fahrzeuge Einzug finden? Diese Fragen sollten von staatlicher Seite möglichst schnell beantwortet werden.

Auf Grund der Studienergebnisse wird in jedem Fall deutlich, dass die Eigenverantwortung des Autofahrers gerade bei Nachtfahrten, wenn sie dann wirklich nicht zu vermeiden sind, besonders gefordert ist. Fahrzeiten über zwei Stunden ohne ausreichende Pause sollten stets vermieden werden.

K. RASCHE =

- J. C. Verster et al.

Prolonged nocturnal driving can be as dangerous as severe alcohol-impaired driving J Sleep Res 20 (2011) 585-588

\section{Lichtenberg-Figuren}

— Eine 19-jährige Frau wurde nach einer Blitzschlag-Verletzung in die Nothilfe gebracht. Ihre große dänische Dogge wurde bei dem Blitzschlag sofort getötet. Die Frau war nach dem Ereignis kurz bewusstlos, fand sich selbst auf dem nassen Boden liegend wieder und konnte Hilfe herbeiholen. Bei der Ankunft im Krankenhaus befand sie sich im Sinusrhythmus, hatte normale Vitalparameter, klagte jedoch über Parästhesien in beiden Beinen. EKG, Troponin I, CK und die Elektrolyte waren unauffällig. Bei der Untersuchung erkannte man mehrere schmerzlose Hautblutungen in der Form eines Farnblattes an der Innenseite beider Beine, die so genannten Lichtenberg-Figuren. Bei der Entlassung

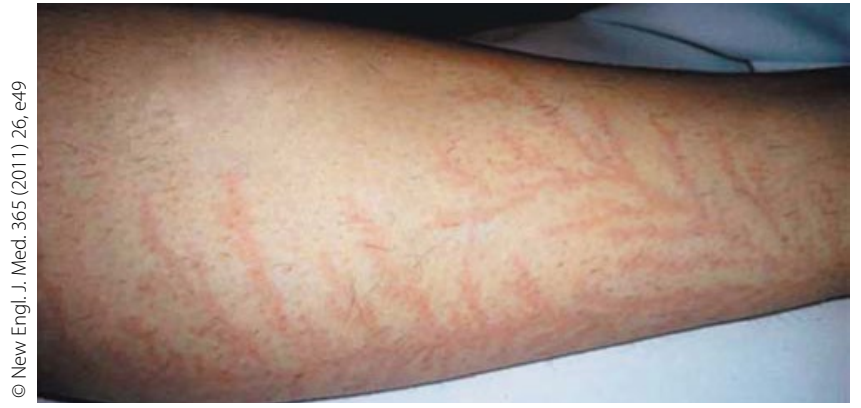

Solche Spuren hinterlässt ein Blitzschlag.

18 Stunden später waren die Hauterscheinungen vollständig verschwunden.

Lichtenberg-Figuren sind baum-, farn- oder sternförmige Muster, die infolge elektrischer Hochspannungsentladungen auf oder in isolierenden Materialien (Dielektrikum) entstehen. Sie sind ladenen Isolatorplatte in seinem Labor entdeckte.

H. S. FÜESSL =

- T. Schmidhauser und A. Azzola (Korres.: tanja.schmidhauser@eoc.ch): Lichtenberg Figures. New Engl. J. Med. 365 (2011) 26, e49 